\title{
ADAPTIVE METHODS FOR DITHERING COLOR IMAGES
}

Lale Akarun

\author{
Boğaziçi University
}

Dept. of Computer Eng.

İstanbul, TURKEY.
Yasemin Yardımcı

\author{
Boğaziçi University, \\ Dept. of Electrical Eng. \\ İstanbul, TURKEY.
}

\author{
A. Enis Çetin \\ Bilkent University, \\ Dept. of Electrical Eng. \\ Ankara, TURKEY.
}

\begin{abstract}
Most color image printing and display devices do not have the capability of reproducing true color images. A common remedy is the use of dithering techniques that exploit the lower sensitivity of the eye to spatial resolution and exchange higher color resolution with lower spatial resolution. In this paper an adaptive error diffusion method is presented. The error diffusion filter coefficients are updated by a normalized LMS type algorithm to prevent textural contours, color impulses and color shifts which are among the the most common side effects of the standard dithering algorithms.
\end{abstract}

\section{INTRODUCTION}

Most color image printing and displaying devices do not have the capability of reproducing true color images [1]. Consequently, the number of colors has to be reduced drastically. A common remedy is the use of dithering techniques that exploit the lower sensitivity of the eye to spatial resolution and exchange higher color resolution with lower spatial resolution. The eye averages the colors in a neighborhood and creates the illusion of more colors. Error diffusion achieves this effect by distributing the error encountered in quantizing a pixel to neighboring pixels, ensuring in effect, that the neighboring pixels are biased in the reverse direction. Some well-known error diffusion filters are Floyd-Steinberg, Stucki, Jarvice, Judice and Ninke [2]-[5].

Common problems that are addressed by dithering are color shift and the appearance of false textural contours. When an image is quantized into a limited number of colors that form a palette, the color of a region is classified into one of the colors in the palette, resulting in a shift which is visible to the eye. A worse-looking defect, however, is the formation of false edges in areas of the image where, there is originally a smooth transition between two colors in the palette. Error diffusion

\footnotetext{
This study is supported by TUBITAK grant EEEAG115
}

solves these two problems in most of the cases: By balancing the quantization errors, it produces an average color shade which is closer to the original color of the region and breaks up the false edges. However, if the given color is close to the boundary of a color cluster and the filter coefficients are not adequately selected, the quantization errors accumulate and eventually, a palette color from a different cluster is produced. This manifests itself as a color impulse which is very disturbing. Another adverse effect of dithering manifests itself around edges: Colors from two sides of the edge are smeared to each other and sharp edges are converted to jagged edges.

In this paper a new adaptive error diffusion technique is proposed to correct these adverse effects: The error diffusion filter coefficients are obtained by minimizing the mean squared error between the average color of the original image and the dithered image. Considering the fact that there may be significant differences in the statistics of different regions in a typical image an LMS type adaptive algorithm is used to solve the minimization problem. This adaptive technique not only produces dithered images in which the average color shade is closest to the original and the occurance of color impulses is greatly reduced but false textural contours do not appear as well.

In Section 2 the adaptive error diffusion algorithm is described. In Section 3 effects of scaling the error diffusion filter coefficients is discussed, and in Section 4 simulation examples are presented.

\section{ADAPTIVE ERROR DIFFUSION ALGORITHM}

Figure 1 shows the block diagram of the error diffusion technique. A color image is represented by $3 \times 1$ vectors $\mathbf{x}_{s}$ for $s=0,1,2, \ldots,\left(N_{1} \times N_{2}\right)-1$. To simplify the equations, double indices denoting the location of the pixels have been replaced by the single index $s$. For an image of size $N_{1} \times N_{2}$ the $\left(n_{1}, n_{2}\right)$-nd pixel corresponds to the index $s=n_{1}+n_{2} N_{1}$. Given the image $\mathbf{x}_{s}$ 


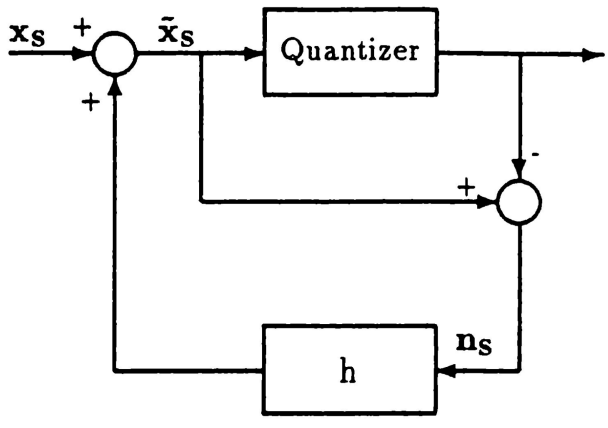

Figure 1: Block diagram of the error diffusion method.

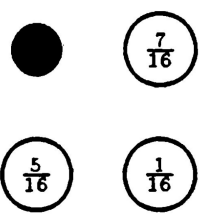

Figure 2: Filter mask of the error diffusion method and the Floyd-Steinberg filter coefficients $(N=4)$.

and a quantizer $Q$, error diffusion works as follows: The quantization error $\mathbf{n}_{\boldsymbol{s}}$ is diffused to neighboring pixels to create a dithered image $\tilde{\mathbf{x}}_{s}$. Then, the dithered image is quantized instead of the original image:

$$
\begin{aligned}
& \tilde{\mathbf{x}}_{s}=\mathbf{x}_{s}+\sum_{k<s} h_{s-k} \mathbf{n}_{k} \\
& \mathbf{n}_{s}=\tilde{\mathbf{x}}_{s}-Q\left(\tilde{\mathbf{x}}_{s}\right)
\end{aligned}
$$

where $k<s$ corresponds to a causal error diffusion mask and the filter coefficients $\mathbf{h}=\left(h_{1} \ldots h_{N}\right)^{T}$ are scalars. In standard Floyd-Steinberg error diffusion method, the filter coefficients $h$ are fixed and independent from the image to be dithered. Figure 2 shows the mask of the Floyd-Steinberg filter. Usually the coefficients are chosen as $\frac{7}{16}, \frac{1}{16}, \frac{5}{16}$ and $\frac{3}{16}$. In our algorithm, the error diffusion filter coefficients $\mathbf{h}=\left(h_{1} \ldots h_{N}\right)^{T}$ are obtained by minimizing the mean squared error between the average color value of the original image and the dithered image. In order to obtain the optimum error diffusion filter coefficients the quantity

$$
E\left\{\left\|\mathbf{x}_{\mathbf{s}}-Q\left(\tilde{\mathbf{x}}_{\mathbf{s}}\right)\right\|^{2}\right\}
$$

is to be minimized with respect to $h_{i}$. This is equivalent to minimizing:

$$
E\left\{\left\|\mathbf{n}_{\mathbf{S}}-\sum_{k<s} h_{\mathbf{s}-k} \mathbf{n}_{\mathbf{k}}\right\|^{2}\right\} .
$$

with respect to the filter coefficients. Differentiating Equation (4) with respect to $h_{i}$ and setting the results to zero, the following set of linear equations are obtained:

$$
E\left\{\mathbf{n}_{s}^{\mathrm{T}} \mathbf{n}_{s-i}\right\}=\sum_{k<s} h_{s-k} E\left\{\mathbf{n}_{k}^{\mathrm{T}} \mathbf{n}_{s-i}\right\}
$$

where $i=1,2, \ldots, N$, the $\mathbf{n}_{s}$ is the quantization error at location $s$. By solving the system of Equations in (5) the error diffusion filter coefficients $\mathbf{h}=$ $\left(h_{1} \ldots h_{N}\right)^{T}$ can be obtained. The covariance coefficients $E\left\{\mathbf{n}_{k}^{\mathrm{T}} \mathbf{n}_{s-i}\right\}$ are not available in practice but they can be estimated from the quantization error statistics of the original image. However this approach does not yield good results because a typical image can not be assumed to be a stationary random field.

Considering the fact that there may be significant differences in the statistics of different regions of a typical image we use an LMS type adaptive algorithm to determine the error diffusion filter coefficients. The key step is to replace the ensemble average with the index average and compute the stochastic gradient vector. For each color component we minimize

$$
e_{\ell}^{2}(s)=\left(n_{s}^{\ell}-\sum_{k<s} h_{s-k}^{\ell} n_{k}^{\ell}\right)^{2}
$$

where $\mathbf{h}^{\ell}$ is the filter coefficient vector for the color component $\ell$. At the index $s$ the filter coefficient vector is updated using the following equation

$$
\mathbf{h}^{\ell}(s+1)=\mathbf{h}^{\ell}(s)-\mu \nabla e_{\ell}(s)^{2}
$$

where $\mu$ is the step size. The gradient is given by

$$
\nabla e_{\ell}^{2}(s)=-2 e_{\ell}(s) \mathbf{z}_{s}^{\ell}
$$

where the vector $\mathbf{z}_{\boldsymbol{s}}^{\ell}$ contains the past quantization errors,

$$
\mathbf{z}_{s}^{\ell}=\left[n_{s-1}^{\ell} \ldots n_{s-N}^{\ell}\right]^{T}
$$

In our simulation studies we employed the so-called normalized LMS update equation [10]:

$$
\mathbf{h}^{\ell}(s)=\mathbf{h}^{\ell}(s)+\mu \frac{e_{\ell}(s) \mathbf{z}_{s}^{\ell}}{\left\|\mathbf{z}_{s}^{\ell}\right\|^{2}}
$$

where the step size parameter $\mu$ is chosen in the interval $(0,2)$ as in the case of the normalized LMS algorithm, and $e_{\ell}(s)$ can be calculated as follows:

$$
e_{\ell}(s)=\left(\tilde{\mathbf{x}}_{s}-Q\left(\tilde{\mathbf{x}}_{s}\right)\right)^{\ell}-\sum_{k<s} h_{s-k}^{\ell} \mathbf{n}_{\mathbf{k}}^{\ell}
$$

In the halftoning of monochrome images for display on bilevel devices, the quantization levels corresponding to the on/off states are at two opposite ends of a 
scale, represented by zero and one. In the error diffusion methods [2]-[5], the filter coefficients are positive numbers that are selected to add up to one. This results in adequate representation of all pixel intensities because the convex hull of the quantization levels contains all possible gray scales. In color error diffusion, this is no longer valid. More quantization levels are required to represent all colors, and common algorithms attempt to place color quantization levels near the center of color clusters [6]. Consequently, some colors in the original image are outside the convex hull of the quantization levels. Error diffusion algorithms whose coefficients are selected as positive constants do not adequately address this problem. When a color outside the convex hull of quantization colors is encountered, this results in the accumulation and ultimate saturation of quantization errors. This manifests itself as a colored impulse. One method described in [6] attemts to avoid this incident by checking the magnitude of the error against a threshold that is proportional to the cluster size for the given color. Our method avoids the saturation of errors altogether: Inspection of Equation (10) reveals that the filter coefficients $\dot{n}_{s}^{\ell}$ can become negative, allowing for recovery from saturation of errors. However, the sum of the magnitudes of the filter coefficients obtained by Equation (10) may exceed one. In such cases we scale the coefficients down so that their sum is one. Effect of scaling the filter coefficients is discussed in Section 3.

Recently, adaptive error diffusion was applied to gray level images in [8] and [9]. Our adaptive algorithm uses a different error measure from [8] and [9] and the resulting filter update equation is simpler.

\section{EFFECTS OF SCALING THE FILTER COEFFICIENTS}

As outlined in Section 2, one of the major problems in the error diffusion of color images is the appearance of color impulses. In many standard algorithms, the error diffusion filter coefficients are defined to sum to one. That corresponds to diffusing all of the error encountered in quantizing a pixel to neighboring pixels. If the coefficients are scaled by a figure that is greater than one, that results in positive feedback, and the results are disastrous. However, if the scaling coefficient is smaller than one, the effect is a cross between error diffusion dithering and doing no dithering at all: If the scaling coefficient is around $90 \%$, doing a little less dithering even improves the appearance in terms of color impulses. This suggests an adaptive algorithm for scaling the error diffusion filter coefficients: In regions where there is little activity, false countours are
Table 1: Subjective Comparison of Dithering Algorithms

\begin{tabular}{|l|l|l|l|}
\hline \multicolumn{4}{|c|}{ Flower (Figure 3) } \\
\hline Algorithm & Texture & Impulses & False Edges \\
\hline No dithering & none & none & heavy \\
F-S [3] & medium & heavy & medium \\
Adaptive & low & none & none \\
\hline \multicolumn{4}{|c|}{ Peppers (Figure 4) } \\
\hline Algorithm & Texture & Impulses & False Edges \\
\hline No dithering & none & none & heavy \\
F-S [3] & low & heavy & medium \\
Adaptive & low & little & little \\
\hline \hline
\end{tabular}

not likely to show up. On the other hand, color impulses are highly noticeable when they occur in those constant regions. Therefore, relaxing error diffusion by spreading only a portion of the error yields superior results in such regions. We adapt the scaling coeffcient based on a measure of activity and change the amount of error diffused. This simple algorithm yields satisfactory results: The appearance of color impulses is eliminated almost completely, and the compromise in terms of the slight increase in the appearance of false contours is outweighed by the overall improvement.

\section{SIMULATION RESULTS AND CONCLUSION}

We compare the results of dithering with the filter mask of Equation (10) with that of the Floyd-Steinberg mask. The color images corresponding to the results are given in Figures 3 and 4 . The input images have been quantized to 16 colors with the median-cut algorithm [7]. It is observed that quantization to 16 colors results in the appearance of false edges. Figures 3a and $4 \mathrm{a}$ are the results of error diffusion with the FloydSteinberg algorithm. It is observed that the application of the Floyd-Steinberg algorithm causes the creation of colored impulses and false textures while alleviating the appearance of false contours. Figures $3 \mathrm{~b}$ and $4 \mathrm{~b}$ show the results of our adaptive algorithm. The appearance of disturbing colored impulses and false textures is minimized, while the false contours are completely eliminated. Table 1 summarizes these subjective comparisons.

In this paper, we developed an adaptive error diffusion method for color images. The adaptation criterion is based on the quantization error statistics. In standard error diffusion methods the filter has a unity gain. We observed that this restriction can be relaxed and the diffusion filter may have a gain slightly less than 

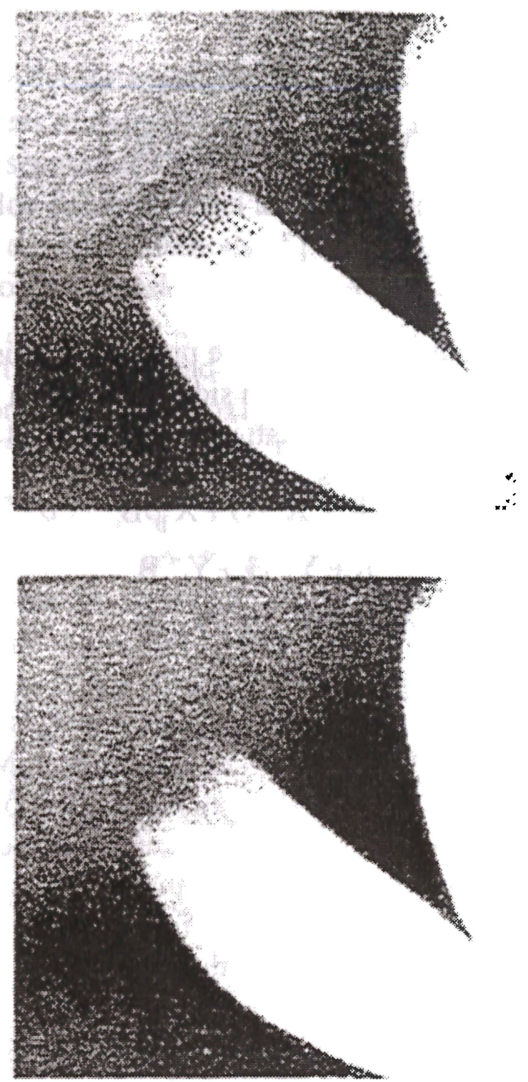

Figure 3: Dithering an image quantized to 16 colors with the median-cut algorithm: (a) Error diffusion with the Floyd-Steinberg filter (b) Error diffusion with the proposed filter

one. This leakage is especially useful to avoid impulses occuring in flat areas of the image.

\section{REFERENCES}

[1] Jan Allebach, "Digital Color Imaging: Bringing Color to the Desktop," IEEE SP-Society Distinguished Lecturer's series: 3. Sinyal Isleme Kurultayz, Goreme, Turkey, May 1995.

[2] R.A. Ulichney, "Dithering with blue noise," Proc. IEEE, vol. 76, pp. 56-79, Jan. 1988.

[3] R.W. Floyd and L. Steinberg, "An adaptive algorithm for spatial grayscale," Proc. SID, vol. 17, no.2, pp. $75-77,1976$.

[4] J.F. Jarvis, C.N. Judice, and W.H. Ninke, "A survey of techniques for the display of continuous tone pictures on bilevel displays," Comput. Graphics, Image Processing, vol. 5, pp. 13-40, 1976.
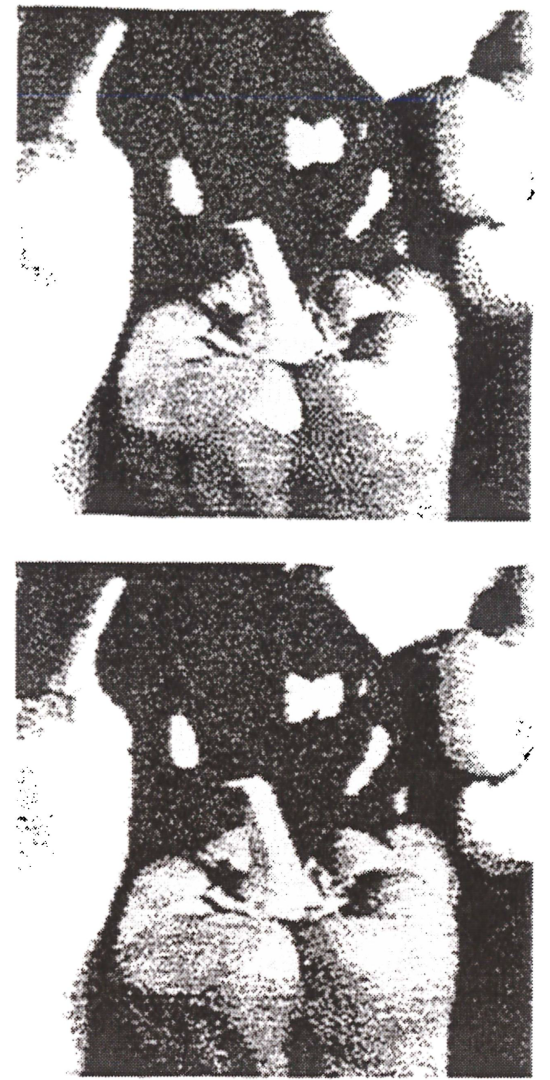

Figure 4: Dithering an image quantized to 16 colors with the median-cut algorithm: (a) Error diffusion with the Floyd-Steinberg filter (b) Error diffusion with the proposed filter

[5] R. Gentile, E. Walowit, Jan Allebach, "Quantization and multilevel halftoning of color images for near-original quality," J. Opt. Soc. Amer. A., vol.7, pp. 1019-1026, 1990.

[6] M.T. Orchard and C.A. Bouman, "Color quantization of images," IEEE Trans. Signal Processing, vol. 39, pp. 2677-2690, Dec. 1991.

[7] P. Heckbert, "Color image quantization for frame buffer display," Computer Graph., vol. 16, no 3, pp. 297-307, July 1982.

[8] R. P. Mironov and R.K. Kunchev, "Adaptive error duffision for image quantisation," Electronics Letters, pp. 2021-2022, vol. 29, November 1993.

[9] P. W. Wong, "Error diffusion with dynamically adjusted kernel," ICASSP'94, pp. V-113-116, 1994.

[10] B. Widrow and S.D. Stearns, Adaptive Signal Processing, Prentice Hall, N.J., 1985. 